\section{PPFL-2195}

$130 / 36.8585$ (2)

$$
D R-C \nabla 92 \cdot 3
$$

PPPL-219.5

$$
I-20167
$$

A HELICAL AXIS STELLARATOR EQUILIBRIUM MODEL

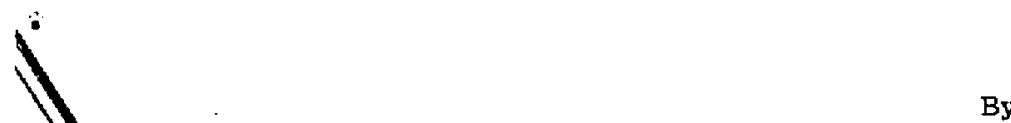

A.E. Koniges and J.L. Johnson

FEBRUARY 1985

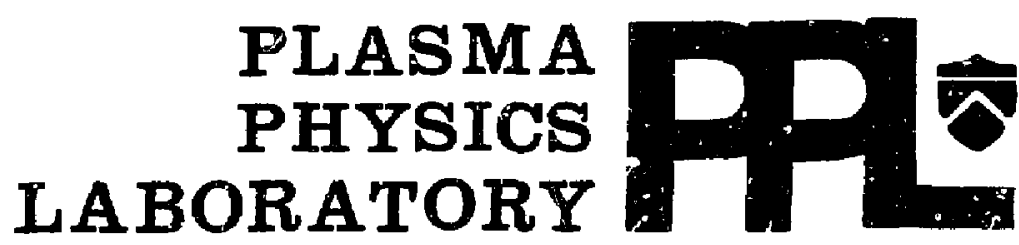

PLASMA PHYSICS

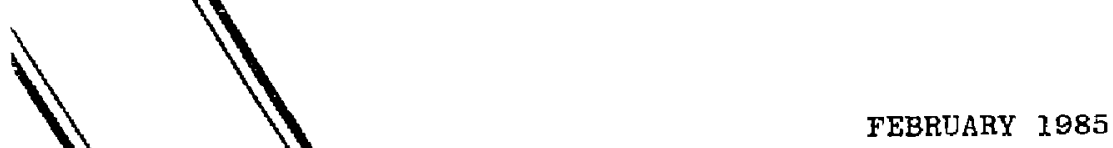

PRINCETON UNIVERSITY

PRINCETON, NEW JERSEY

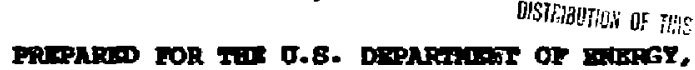

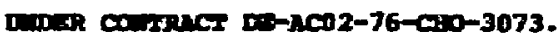


This repon wa prepand is an accont of work poosored by es apeacy of the United Siatea Government. Neither the United Sinte Government nor abj seacy theroof, nor uny of their

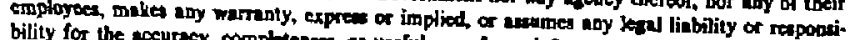
process dieclased of completenes, or uerulacs of any information, apparalus, product, of ptocess disclosed, of represents that its ue womid not infringe privately owned righte. Refermaufecturer, or atherifs conmercial produch, procese, or ervice by trade name, trademerk. madufacturer, or oftermise does bot aecewarily contitute or imply its endersement, recomand opinions of United Stales Government or any agency thereof. The view Uniled States Goremment or any apency ihereof.

\title{
A Helical Axis Stellarator Equilibrium Model
}

\author{
A. E. Koniges ${ }^{1}$ \\ Plasma Physics Laboratory and Program in Applied Mathematics \\ Princeton University, Princeton, NJ 08544 \\ J. L. Johnson ${ }^{2}$ \\ Plasma Physics Laboratory \\ Princeton University, Princeton, NJ 08544
}

An asymptotic model is developed to study MHD equilibria in toroidal systems with a helical maguetic axis. Using a characteristic coordinate system baged on the vacuum field lines, the equilibrivm problem is reduced to a twodimensional generalized partiai differential equation of the Grad-Shafranov type. A stellarator-expansion free-boundary equilibriun code is modified to solve the helical-axis equations. The expansion model is used to predict the equilibitum properties of Asperators NP-3 and NP-4. Numerically determined fux surfaces, magnetic well, trangform, and shear are presented. The equilibria ghow a toroidal Shafranov shift.

\footnotetext{
1present addrea: Magnetic Fuaion Energy Computer Cet, ter, Lawerence Livermore Laboratory, Livermore, Culiforeiz 94550

${ }^{2}$ On laun fram Wetinghouse Reaearch and Development Center
} 


\section{Introduction}

The concept of a toroidal stellarator with a nonplanar magnetic axis origivated early in the fusion program. 1.2 The recent favorable outlook for the stellarator approach ${ }^{3}$ has led to the exploration of new helical-axis configurations which offer hopes for high- $\beta$ confinement. To better understand the confinement properties of such devices, methods for analyzing configurations with nonplanar axes mugt be developed.

The complex topology of stellarator geometries makes theoretical MHD equiJjbrium analysis complicated. Since there is no igoorable coordinate, it is not possible to derive an exact Grad-Shafranov-type equation for the equilibrium. Numerical equjlibrium computation using three-dimessional computer codes has been successful for botb planar and nonplanar devices." However, threedimensional calculations are limited to relatively coarse mesh size by the speed and memory of preseut computers, and in their ability to compute free-boundary equilibria Approximate metbods based on as expansion about a prescribed magnetic axis ${ }^{7,8,9}$ have been used for the analysis of nonplanar toroidal systems. These methods are limited by dificulties in the incorporation of boundary conditions and by restrictions on the functional form of the assumed curreat and pressure profiles. There bas been considerable success in using appropriate asymptotic expansions to reduce both the equilibrium and stability problems to simpler ones in two dingensions where a generalized partial diferential equation of the Grad-Shafranov type can be obtained. For tb- conventional (planar axis) stellarator, the stejlarator expangion of Greene and sohnson ${ }^{10}$ and its numerica! implemention for solving free-boundary equilibria ${ }^{11}$ is cirrently a valuable tooi 
for desigo of devices and interpretation of experimental results. However, the assumptions made in the standard expansion yield a lowest-order configuration which has axial aymmetry, and limit its applicability to systems with nearly planar axes.

In tbis paper, we develop all asymptotic model for MHD equilibria of systems with a belical magnetic axis of large radius. We interpret the nonplanar behavior of the magnetic axis as being associated with an $\ell=1$ stellarator feld, and use an ordering which is basically an extengion of the standard stellarator expansion. ${ }^{10}$ The majur modification is that the wavelength of the primary helical field is treated as large with reapect to the plasma radius, whereas in the standard stellarator expansion, these quantities are of the same order. Following the introduetion of two length scales in the torojdal direction, we transform to a characteristic coordinate system based on the lowest-order vacuum feld lines, which follow a belical path. This enables us to implement the standard asymptotic procedure ${ }^{12}$ of integrating the lowest-onder equationg and removing secular terms in higher order analytically. The equilibrium problem is reduced to the solution of a two-dimensional Grad-Shafranov-like equation. The problem is solved using aumerical techniques developed for the standard stellarator expansion. In thix paper, we study the equilibrium properties of a large-aspectratio, low- $\beta, \ell=1$ stellarator, altbough the formalism can be extended to treat systems with more complicated fields.

We present the governiag equations in Sec. II. In Sec. III, we discuss the geometry, the vacuum fields, and the asymptotic ordering. In Sec. IV, we introduce the characteristic coordinates and reduce the problem to a two-dimensional 
formulation. Some equilibrium quantities are calculated in Sec. V. The boundary conditions are presented in Sec. VI. Applications of the model are given in Sec. VII.

\section{Banic Equations}

The static equilibrium configuration is degcribed by the MHD equations,

$$
\begin{gathered}
\nabla P=\mathrm{J} \times \mathrm{B}, \\
\nabla \cdot \mathbf{B}=\mathbf{0} \\
\nabla \times \mathbf{B}=\mathbf{J} .
\end{gathered}
$$

It is convenient to cast these equations into the form of two magnetic differential equations and a vector Poisson equation. We agsume the configuration has nested topologicelly toroidal magnetic surfaces which we label as the level surfaces of a function $\bar{\Psi}$, and prescribe a functional dependence for the scalar pressure, $P=P(\Psi)$. (In Section $V, \Psi$ is chosen to be proportional to the poloidal fux.) Thes Eq. (1) yields

$$
\mathbf{B} \cdot \nabla \Psi=0
$$

We write the total magnetic field as

$$
\mathbf{B}=\mathbf{B}_{\mathbf{r a c}}+\mathbf{B}^{\alpha}+\mathbf{B}^{\beta},
$$

where $B_{\mathrm{vac}}$ is the vacuum Geld, and $\mathrm{B}^{\circ}$ and $\mathrm{B}^{\beta}$ are the fieids induced by currents parallel and perpendicular to the field, respectively. Following standard 
procedure, we split the current dersity into perpendicular and parallel components,

$$
\mathbf{J}=\mathbf{J}_{\perp}+\boldsymbol{\sigma} \mathbf{B},
$$

where, from Eq. (1),

$$
\mathbf{J}_{\perp}=\frac{\mathbf{B} \times \nabla P}{\mathbf{B}^{2}}
$$

and

$$
\sigma \equiv \frac{\mathbf{J} \cdot \mathbf{B}}{\mathbf{B}^{2}}
$$

The fields induced by these currents can be divided into components $\mathbf{B}^{\beta}$ and $\mathbf{B}^{\sigma}$, with

$$
\nabla \times \mathbf{B}^{\beta}=\mathbf{J}_{\perp}
$$

and

$$
\nabla \times \mathbf{B}^{\alpha}=\boldsymbol{\sigma B} .
$$

It is convenjent to write $\mathrm{B}^{\sigma}$ in terms of a vector potential,

$$
\mathbf{B}^{\sigma}=\nabla \times \mathbf{A}^{\sigma},
$$

and, choosing the gauge with $\nabla \cdot \mathbf{A}^{\sigma}=0$, we have

$$
\nabla^{2} A^{\sigma}=\sigma \mathbf{B}
$$

A magnetic differential equation for $\sigma$,

$$
\mathrm{B} \cdot \nabla \sigma=\frac{\mathrm{B} \times \nabla P \cdot \nabla B^{2}}{B^{4}},
$$

is obtained from the condition that $\mathbf{J}$ be divergeace-free.

Equations (4), (5), and (6) will be used in conjunction with the expansion developed in the next section to form a generalized partial differential equation for the lowest-crder equilibrium configuration. 


\section{Vacuum Field and Expangion Ordaring}

The model geometry is a tube with a helical axis; the helical axis is inscribed in a cylinder which is bent into a torus with a large radius oi curvature. The radius of the cylinder defines the belical radius. I'he belix is periodic over a length $2 \pi / h$. The vacu'm field, $B_{\text {vac }}$, is the superposition of a toroidal field, $\mathbf{B}_{0}$, which is constant to lowest order, and an $\ell=1$ helical stellarator field, $\mathbf{B}_{\mathrm{H}}$. The toroidal currature is introduced by solving for the vacuum field potential in a pseudo-ior.jidal coordinate system. This orthogonal coordinate system is defiaed by giving the metric

$$
d d^{2}=d r^{2}+r^{2} d \omega^{2}+\left(1+\frac{r}{\pi} \cos \omega\right)^{2} d z^{2},
$$

where $(r, \omega, z)$ is a local cylindrical system centered on the toroidal axis, and $\boldsymbol{R}$ is the major radius of the corus. The toroidal field $\mathbf{B}_{0}$ is written in tr.5ms of a scalar potential proportionel to $z$, and curvature terms associatud with this field enter through expansion of the gradient opesator in powers of the curvature. The scalar poteatjal for the helical field $\mathbf{B}_{\mathbf{H}}$ is a solution of Laplare's equation in pseudo-toroidal coordinates, which is helically symmetric to lowest order.

The assumptions motivating the ordering are that the magnitude of the helical field is small with respect to the toroidal field and that the wavelength of the helical field is sufficiestly long that the distortion of the magnetic axis from being straight (i.e., thehelical radius) is of the same order as the plasma radius. This leads to an orderiug in which the plasma radius is small with respect to the helical wavelength, which in turn is small with respect to the toroidal circumference. To incorporate these into the framework of a siellarator 
expansion, we introduce two length scales in $z$, a rapid variation that is periodic over one belical period denoted by $t$, and a slow variation all the way around the torus denoted by $\varsigma$. We assume that all physical scalara are periodic functions of $t$ as well as 5 . We introduce dimensioniess variables

$$
x=\frac{r}{a} \cos \omega, \quad y=\frac{r}{a} \sin \omega, \quad t=h z, \quad s=\frac{z}{a},
$$

where $a$ is the minot radius of the plasma. Then, standard multiple-scale anatysis gives

$$
a \frac{\partial}{\partial z}=\frac{\partial}{\partial \zeta}+\gamma \frac{\partial}{\partial t}+\cdots
$$

where $\gamma \equiv h a \ll 1$.

In what follows, we work in a get of units witk lengths normalized to the plasma radius a and magnetic fields nermalized to the toroidal field strength $\left|\mathbf{B}_{0}\right|$. We define a basic expansion parameter $\delta$, measuring the rejative strength of the helical Geld with respect to the toroidal field. The geometric parameters are ordered in terms of $\delta$ as

$$
\delta \sim \gamma \sim \iota^{\frac{1}{2}},
$$

where $\delta \equiv\left|\mathbf{B}_{\mathrm{H}}\right| /\left|\mathbf{B}_{0}\right|$, and $\epsilon \equiv a / R$ is the inverse aspect ratio. Then, the total vasuum field $B_{\text {vec }}$ is expreaged in terms of a sealar potential, $\mathbf{B}_{\mathrm{vac}}=\nabla \Phi$, with

$$
\begin{gathered}
\Phi=\varsigma+\gamma \Delta(y \cos t-x \sin t)+\frac{\gamma^{3} \Delta}{8}\left(x^{2}+y^{2}\right)(y \cos t-x \sin t) \\
+\frac{\gamma \epsilon \Delta \rho^{2}}{4} \sin t+O\left(\delta^{4}\right)
\end{gathered}
$$

where $\Delta \equiv \delta / \gamma=O(1)$. The $\gamma \in \Delta$ term arises from the coupling betwcen helicity and toroidicity in solving Laplace's equation for the helical feld. The ordering of $\gamma$ with respect to $\delta$ in chosen to ensure that the cylindrical radius of the helix 
be of the same order as the plasma radius, and the ordrring of $\epsilon$ is chosen go that all three parameters enter the expression for the lowest-order flux surfaces.

The helical nature of the magnetic field lines is best, observed by considering the field-line equations,

$$
\frac{d x}{B_{x}}=\frac{d y}{B_{y}}=\frac{d t(1+\epsilon x)}{\gamma B_{z}}
$$

To lowest order, we have

$$
\begin{gathered}
\frac{d x}{d t}=-\frac{\delta}{\gamma} \sin t, \quad x=x_{0}-\frac{\delta}{\gamma}+\frac{\delta}{\gamma} \cos t, \\
\frac{d y}{d t}=\frac{\delta}{\gamma} \cos t, \quad y=y_{0}+\frac{\delta}{\gamma} \sin t_{1}
\end{gathered}
$$

where the constants $x_{0}$ and $y_{0}$ are the intercepts of the field lines with the $t=0$ gurface. (In the standard stellarator expansion where $\gamma \sim 1$, the field Fines follow a circular path around the torus to lowest order.) Carrying the integration of these equations for the vacuum field lines to higher order shows that the rotational transform is $\iota=\delta^{2} / 2$ per helical period. (Our definition of $\iota$ differs from that of Greene and Johngon ${ }^{10}$ by a factor of $2 \pi$.) Since the total oumber si helical periods is $\gamma / \epsilon$, the trangform over a complete circuit of the torus is of order $\delta$.

The effects of plasma pressure and current on the equilibrium enter through the fields $\mathrm{B}^{\beta}$ and $\mathrm{B}^{\alpha}$ introduced in the previous section. We choose the ordering of tine pressure so that it affects the lowest-order magnetic surfaces. It will be shown in the Dext section that, given the ordering of the vacuum field parameters $\delta, \tau$, and $\epsilon$, the thisd-order components of $B_{\perp}$ affect the determisation of $\Psi$ to lowest order through. Eq. (4). This Gxes the sjze of $\mathbf{B}^{\sigma}$, and we can determine 
the order of $B^{\beta}$ from Eq. (6). Thus we take

$$
\frac{B^{\alpha}}{B_{0}} \sim \delta^{3}, \quad \frac{B^{\beta}}{B_{0}} \sim \delta^{3} .
$$

This is a maximal balance ordering in the sense that all the ordered physical quantities enter into the determination of the lowest-order magnetic surfaces.

\section{Integration}

In this section, we use the asymptotic expansion to simplify and integrate the equations introduced in Sec. II. Since B is a constant field in the toroidal direction in lowest order, we can simplisy the lowest-order vector potential to one component $\mathbf{A}^{\sigma}=A(x, y, t) \hat{z}+\cdots$. (Here, $\hat{z}$ is the unit vector in the toroidal dirention.) Then Eq. (5) reduces to an inhomogeneous scalar equation

$$
\nabla_{\perp}^{2} A=\sigma
$$

where $\nabla_{\perp}^{2} \equiv \partial^{2} / \partial x^{2}+\partial^{2} / \partial y^{2}$.

The two magnetic differential equations (4) and (6) can be integrated order by order. An efficacious transformation to a coondinate system baged on the characteristics of the operator B $\nabla$ enables us to formulate the equilibrium problem in two dimensions.

We start with Eq. (4) for the magnetic surfaces and expand

$$
\Psi(x, y, \mathfrak{t}, s)=\Psi^{(0)}+\Psi^{(1)}+\ldots,
$$

where superscripts denote order with respect to $\delta$. The equation in lowest order is

$$
\frac{\partial \Psi^{(0)}}{\partial \varsigma}=0
$$


and periodicity requires that

$$
\Psi^{(0)}=\Psi^{(0)}(x, y, t),
$$

which means $\Psi^{(0)}$ is allowed to vary in the toroidal ditection only over the short (belical) period. It can be shown that all $\zeta$ dependence is similarly eliminated order by order. The argument follows from Kruskal's theorem of phase independence. ${ }^{13}$

In the next order,

$$
L\left[\Psi^{(0)}\right] \equiv \frac{\partial \Psi^{(0)}}{\partial t}-\Delta \sin t \frac{\partial \Psi^{(0)}}{\partial x}+\Delta \cos t \frac{\partial \Psi^{(0)}}{\partial y}=0 .
$$

This first-order linear equation is conveniently solved by integrating the characteristic equations,

$$
\frac{d t}{1}=\frac{-d x}{\Delta \sin t}=\frac{d y}{\Delta \cos t} ; \quad d \Psi^{(0)}=0 .
$$

Solying simultaneously yields the general solution,

$$
\Psi^{(0)}=\Psi^{(0)}\left(C_{1}, C_{2}\right)
$$

w'sere

$$
C_{1} \equiv x-\Delta \cos t, \quad C_{2} \equiv y-\Delta \sin i
$$

Similarly, in second order, we find

$$
\Psi^{(1)}=\Psi^{(1)}\left\{C_{1}, C_{7}\right\}
$$

The functional form of $\Psi^{(0)}$ is determined by the third-order equation,

$$
L\left[\Psi^{(2)}\right]=-\frac{B_{x}^{[3]}}{\gamma} \frac{\partial \Psi^{(0)}}{\partial x}-\frac{B_{y}^{[3)}}{\gamma} \frac{\partial \Psi^{(0)}}{\partial y}+[\gamma \delta(y \sin t+z \cos t)+z \in x] \frac{\partial \Psi^{(0)}}{\partial t}
$$


where $B_{z}^{(3)}$ and $B_{y}^{(3)}$ are the thjrd-order components of the full niagnetic field. The general golution for $y^{(2)}$ can be obtained by trangforming to a characteristic coordinate system $\left(C_{1}, C\right.$ ? where $C_{1}$ and $C_{2}$ are given in Eq. (14) and $\hat{t}=t$. Note that in this coordinate system, the operator $L\left[\psi^{(3)}\right]$ of $E_{\mathrm{y}}$ (12) becomes $\partial \Psi^{(2)} / \partial \hat{t}$. The condition that $\Psi^{(2)}$ be periodic over a helical period,

$$
\int_{0}^{2 \pi} \frac{\partial \Psi(2)}{\partial \hat{t}} d \hat{t}=0
$$

reduces to a fist-order partial differencial equation which determines the furc. tional form of $w^{(0)}$, namely

$$
\frac{\partial \Psi^{(0)}}{\partial C_{1}}\left\lceil\frac{\gamma^{l} \Delta^{2}}{2} C_{2}+\frac{1}{\gamma} \frac{\partial \bar{A}}{\partial C_{2}}\right]-\frac{\partial \Psi^{(0)}}{\partial C_{2}}\left[\frac{\gamma^{2} \Delta^{2}}{2} C_{1}+\frac{5 \epsilon \Delta^{2}}{\gamma}+\frac{1}{\gamma} \frac{\partial \bar{A}}{\partial C_{1}}\right]=0 .
$$

Here, barred quantities are averages over the helical period,

$$
\bar{A}\left(C_{1}, C_{2}\right) \equiv \frac{1}{2} \pi \int_{0}^{2 \pi} A\left(C_{1}, C_{2}, \hat{t}\right) d \dot{t}
$$

Integration of Eq. (15) shows that $\Psi^{(0)}$ is a iupction of

$$
C_{1}^{2}+C_{1}^{2}+\frac{56}{\gamma^{2}} C_{1}+\frac{4 \bar{A}}{\delta^{2} \gamma}
$$

We choose it to be a linear function in order that $\Psi^{(0)}$ be proportional to the poloidal flux which is calculated in the next section. In particular. we write

$$
\Psi^{(\mathfrak{a})}=C_{1}^{2}+\sigma_{1}^{2}+\frac{5 \epsilon}{\gamma^{2}} C_{1}+d^{2}+\frac{4 \bar{A}}{\delta^{2} \gamma}
$$

where

$$
d \equiv \frac{5 e}{2 \gamma^{3}} .
$$

There is no lass of generality in choosing $\psi^{(\mathrm{D})}$ to be pronortional to the poloidal flux. This ig iust the freedom to label surfaces in a particular way. 
Integration of Eq. (6), the magnetic differential equation for $\sigma$, is similarly expedited by working in the characteristic coordinate systen, since the operator B $\cdot \nabla$ is the same in both cases. With $\nabla P(\Psi)=P^{\prime}\left(\Psi^{(0)}\right) \nabla \Psi^{(0)}+\ldots$, Eq. (6) becomes

$$
\mathbf{B} \cdot \nabla \sigma=P^{\prime}\left(\Psi^{(0)}\right)\left[(2 \gamma \delta \cos t+2 \epsilon) \frac{\partial \Psi^{(0)}}{\partial C_{2}}+2 \gamma \delta \sin t \frac{\partial \Psi^{(0)}}{\partial C_{2}}+O\left(\delta^{3}\right)\right] .
$$

Proceeding as before, we find in fourth order

$$
\sigma^{(3)}=\sigma^{(3)}\left(C_{1}, C_{2}\right) \text {. }
$$

Again, the functional form of $\sigma$ is determined by the periodicity constraint in higher order,

$$
\int_{0}^{2 \pi} \frac{\partial \sigma^{(3)}}{\partial \hat{t}} d \hat{t}=0
$$

which yields

$$
\begin{aligned}
\frac{\partial \sigma^{(3)}}{\partial C_{1}}\left[\frac{\gamma^{2} \Delta^{2}}{2} C_{2}+\frac{1}{\gamma} \frac{\partial \bar{A}}{\partial C_{2}}\right] & -\frac{\partial \sigma^{(3)}}{\partial C_{i}}\left[\frac{\gamma^{2} \Delta^{2}}{2} C_{2}+\frac{5 \epsilon \Delta^{2}}{\gamma}+\frac{1}{\gamma} \frac{\partial \bar{A}}{\partial C_{1}}\right] \\
+ & \frac{2 \epsilon}{\gamma} \cdot P^{\prime}\left(\Psi^{(0)}\right) \frac{\partial \mathcal{X}^{(U)}}{\partial C_{2}}=0 .
\end{aligned}
$$

Integration gives

$$
\sigma^{(3)}=\frac{-8 E}{\gamma^{\delta^{2}}} P^{\prime \prime}\left(\Psi^{(0)}\right) C_{1}+F\left(\Psi^{(0)}\right),
$$

where $F$ is an arbitrary function. The freedom to specify $F$ allows us to salculate equilibria with zero net current.

Finally, we consider Eq. (10) for $A$. Since to lowest-order $\sigma$ is a function of $C_{1}$ and $C_{2}$ only, we take $A=\bar{A}$. We express Eq. (10) in the characteristic coordinate system as

$$
\frac{\partial^{2} A}{\partial C_{1}^{2}}+\frac{\partial^{2} A}{\partial C_{2}^{2}} \approx \sigma^{(3)}\left(C_{1}, C_{2}\right)
$$


The coupled set, Eqs. (16), (17), and (18), can be rumbined to yield a generalized partial differential equation of the Grad-Shafranov type, similar to that obtained uging the standard stellarator expansion. ${ }^{10}$ In Sec. VI, we specify the surface functions $P^{\prime}\left(\Psi^{(0)}\right)$ and $F\left(\Psi^{(2)}\right)$ of Eq. (17) and discusa the boundary canditions fc: the system.

\section{Equilibrium Quantities}

The equilibrium configuration is characterized by the magnetic surface function $\Psi$, given to loweat order by $\mathrm{Eq}$. (16). In the vacu um limit, we have circular fux surfaces with fixed radius $\Psi^{\left(0^{\circ}\right.} \frac{1}{2}$, centered on a helical magnetic axis given by $z=\Delta \cos t-d$, and $y=\Delta \sin t$, where $(x, y, t)$ are the normalized pseutotoroidal coordinates. Toroidal effects enter these lowest-order vacuum surfaces since the constant shift $d=5 \varepsilon / 2 \gamma^{2}$ is proportional to the inverse aspect ratic. The effect of current in the plasma enters through the stream function $A\left(C_{1}, C_{2}\right)$.

It is convenient to calculate equilibrium quantities in a $(\rho, \theta, \hat{t})$ coordinate system with origin centered on the racuum magaetic axis,

$$
p=\left\{\left(C_{1}+d\right)^{2}+C_{2}^{2}\right]^{3}, \quad \theta=\tan ^{-1}\left[C_{2} /\left(C_{1}+d\right)\right], \quad i=t .
$$

where $C_{1}$ and $C_{3}$ are defined in Eq. (14). Then, the angular coordinate function $\theta$ increases by $2 \pi$ during one loop around the magnetic axis the short way, and the poloidal flux per helical period is ${ }^{\text {:4 }}$

$$
\Psi_{\mathrm{pol}}=\frac{1}{2 \pi} \int_{V} b^{\theta} d r
$$

Here, $V$ is the volume enclosed by the surface in question, and the superscript 
on $b$ denotes a contravariant component of the field $\mathbf{B}$, namely

$b^{\theta}=\frac{-\sin \theta}{\rho}\left[B_{x}-\Delta \cos \hat{t}\left(\frac{\gamma B_{x}}{1+\epsilon x}\right)\right]+\frac{\cos \theta}{\rho}\left[B_{y}+\Delta \sin \hat{t}\left(\frac{\gamma B_{x}}{1+\epsilon x}\right)\right]+O\left(\delta^{5}\right)$.

Also,

$$
d \tau=\frac{(1+\epsilon x)}{\gamma} \rho d \rho d \theta d \hat{t}, \quad x=\rho \cos \theta-\Delta \cos \hat{t}-d .
$$

Evaluating the integral over $\hat{t}$ and doing some manipulating yields

$$
\Psi_{p o l}=\int_{0}^{2 \pi} d \theta \int_{0}^{\rho\left(\Psi^{(\theta)}, \theta\right)} \frac{\delta^{2}}{4} \frac{\partial \Psi^{(0)}(\rho, \theta)}{\partial \rho} d \rho=\frac{\pi \delta^{2}}{2} \Psi^{(0)}
$$

where we have retained terms through third order in $\delta$.

The toroidal Hux is given by ${ }^{14}$

$$
\begin{aligned}
\Psi_{\text {tor }} & =\frac{1}{2 \pi} \int b^{t} d t \\
& =\int_{0}^{2 \pi} d \theta \int_{0}^{\rho\left(\sigma^{(0)}, 0\right)}\left(1-\epsilon(\rho \cos \theta-d)-\gamma^{2} \Delta^{3}\right) \rho d \rho+O\left(\delta^{3}\right) .
\end{aligned}
$$

In lowest order where the toroidal feld is constant, the toroidal flux is the crosssectional area enclosed by a given flux surface $\Psi(0)$. This can be written in a Gux coordinate system as

$$
\Psi_{t o r}=\int_{0}^{\Psi^{(0)}} d \Psi^{(0)} \int \frac{d l}{|\nabla \Psi|}
$$

where $d l^{2}=d \rho^{2}+\rho^{2} d \theta^{2}$. The rotational trangform obtained in this manner,

$$
\iota \equiv \frac{d \Psi_{\text {pol }}}{d \Psi_{\text {tor }}}=\pi \frac{\delta^{2}}{2}\left[\int \frac{d l}{|\nabla \Psi|}\right]^{-1}
$$

agrees in the vacuum limit with the transform obtained by integrating the vacuum field-line equations,

$$
\iota_{\mathrm{vac}}=\frac{\delta^{2}}{2}
$$


The volume enclosed by a given flux surface,

$$
V=\frac{1}{\gamma} \int d r=\int_{0}^{2 \pi} d i \int_{0}^{2 \pi} d \theta \int_{0}^{\rho\left(W^{(0)}, \theta\right)} \rho d \rho(1+c(\rho \cos \theta-d))+O\left(\delta^{4}\right),
$$

can be used to calculate the magnetic well, which provides a stability estimate. By adding and subtracting $b^{2}(1+c(\rho \cos \theta-d))$ from the integrand, ${ }^{10}$ we can write $V$ in terms of the toroidal flux and a Alux surface integral;

$$
V=\frac{2 \pi}{\gamma} \Psi_{\text {tor }}+\frac{1}{\gamma} \int d \Psi^{(0)} \int \frac{d l}{|\nabla \Psi|}\left[2 \epsilon(\rho \cos \theta-d)+\gamma^{2} \Delta^{2}\right]+O\left(\delta^{1}\right) .
$$

'This form : $s$ useful for computing the well $V^{\prime \prime}\left(\Psi_{\text {tor }}\right)$, where the prime denotes derivative with respect to the argument, since the potentially stabilizing iategral term can be computed by numerically integrating terms of finite order. To the order of this calculation, $V^{\prime \prime}\left(\Psi_{\text {tor }}\right)=0$ in the vacusm limit. We note that due to the chosen ordering, this result does not contain a tern associated with the d:amagretic currents that maintain the plasma pressure, as was the case in the standard stellarator expansion. ${ }^{10}$

\section{Boundary Conditions and Numerical Solutiona}

In this section, we pose two boundary-value problems for Eq. (18). The freeboundary problem is analogous to those solved by standard iterative stellarator equilibrium codes, and we have modified a version of PEST ${ }^{11,15}$ to solve these helical-axis equations. The details of these modifications are given eisewhere. ${ }^{18}$

\section{A. Free-Boundary Problem}

Consider a plasma surrounded by a vacuum, so that $\nabla_{\perp}^{2} A=0$ in the vacuum and $\nabla_{\perp}^{2} A=\sigma$ in the plasma. A free-boundary problem is formulated in 
the following way. ${ }^{11,15}$ Using an initial guess for $\sigma$ and $\Psi^{(0)}$, we numerically determine the Dirichlet conditian $\left.A\right|_{\text {boundary }}$ of the computational domain by integrating the product of $\sigma$ and the free-space Green's function $G\left(x_{B}, \mid x_{p},\right)$ for the two-dimengional Laplacian operator,

$$
G\left(r_{B} \mid r_{P}\right)=-2 \log \left(\frac{\left|\mathbf{r}_{B}-\mathbf{r}_{P}\right|}{\left|\mathbf{r}_{P}\right|}\right)
$$

over the plasma region. Here, $\mathbf{r}_{7}$ is a point on the boundary, and $\mathbf{r}_{P}$ is a point in the plasma. This is equivalent to the assumption that the plasma itself is the only current source, and that the influence of the current source drops to zero far from the plasma. We note that it is straightforward to modify the Green's function formulation to include the effects of exterual curreat sources. ${ }^{10}$ Given

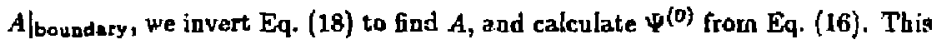
yields a new value for $\sigma$ from Eq. (17) and the procedure is repeated until a self-consistenk solution is obtained.

In general, two surjace functions must be given in order to specify the equilibrium completely. ${ }^{14}$ The pressure is preacribed by

$$
P=P_{0}\left(\frac{\Psi^{(0)}{ }_{\text {lim }}-\Psi^{(0)}}{\Psi_{\text {lim }}^{(0)}-\Psi^{(0)}}\right)_{\text {lnio }}^{k},
$$

where $\Psi^{(0)}$ tim is the value at a limiter which defines the plasma boundary in the vacuum limit and $\Psi^{(0)}$ min is the minimum value of $\Psi(0)$ on the gid. $A$ common choice is $k=2$. The free function $F\left(\Psi^{(0)}\right)$ in Eq. (17) is iteratively determined such that the pure stellarator condition is satisfied, i.e., no net toroida' current on each surface. Thus,

$$
F\left(\Psi^{(0)}\right\}=\frac{B c}{\gamma^{2}} P^{\prime}\left(\Psi^{(0)}\right) \int C_{1} \frac{\| l}{\left|\nabla \Psi^{(0)}\right|}\left[\int \frac{d l}{\left|\nabla \Psi^{(0)}\right|}\right]^{-1} .
$$


We note that we can add to this an additional function of $\Psi^{(0)}$ in order to model equilibria with net toroidal current.

\section{B. Fixed-Boundary Problem}

The fixed-bourdary problem is equivalent to surrounding the plasma with a perfectly conducting shell. (Often, the shape of the shell is chosen to coincide with a vacuum surface.) If the conducting shell's cross section is a circle in $\left(\mathcal{C}_{1}, C_{2}\right)$ space, it is possible to write thr: solution in tlosed form as an integral equation. Hence, we solve $\nabla_{\perp}^{2} A=\sigma$ with $\Psi^{(0)}=$ constant at $\rho=1$. The Greea's function for this problem can be obtained by confortnally mapping the relevant balf-plane Green's function into the unit disk, yielding ${ }^{1 T}$

$$
G(\rho, \theta \mid r, s)=\frac{1}{4 \pi} \log \left[\frac{\rho^{2}-2 \rho r \cos (\theta-\theta)+r^{2}}{1-2 \rho r \cos (\theta-\theta)+p^{2} r^{2}}\right] \quad(\rho \leq 1) .
$$

Then,

$$
\Psi^{(0)}(\rho, \theta)=\int_{d i s k} r d r d a\left[4\left(1+\frac{\sigma\left(\Psi^{(0)}\right)}{\delta^{3} \gamma}\right) G\left(\rho, \theta \mid r_{+} s\right)\right]
$$

where we bave uged the relation,

$$
\nabla^{2} \Psi^{(0)}=4+\frac{4}{\delta^{2} \gamma} \nabla^{2} A
$$

Again, $P\left(\Psi^{(0)}\right)$ is a specifed function, and $F\left(\Psi^{(0)}\right)$ can be determined iteratively to satisfy a condition on the net current.

\section{Applications}

We use the telical-axis stellarator expansion to model two stellarators at Tohoku University, ${ }^{18,19}$ Asperators NP-3 and NP-4. Each of these devices has 
a helical magnetic axis which closes upon itself following one circuit in the toroidal direction. The primary magnetic field is produced by circular solenoids centered on a helical axis. This helical feld should be adequately modeled uging the $\ell=1$ potential of the belical-axis stellaratoc expansion. Device parameters for NP-3 and NP-4, together with the appropriate parameters for the expansion, are given in Table I. ${ }^{18,19}$

The helical-axis stellarator expangion yields gux surfaces which are nested on a primarily belical axis. The toroidal dependence of the configuration is depicted in Fig. 1(a), which shows the surfaces at three different $t$-planes for the NP-3 deyice $\because$ ith an average beta $\langle\beta\rangle=34 \%$. The large cross marks the center of the pseudo-tonoidal coordinates $(x, y, t)$, i.e., the center of the $\ell=1$ feld. The model predicts a toroidal Shafranov shift, which introduces both shear and a magnetic well. The shear is evidenced in Fig. $\mathbf{l}(\mathbf{b})$, since the rotational transform is sot constant. Figure $1(c)$ shows the well, since $V^{\prime}\left(\Psi_{t o r}\right)$ is a monotonically decreasing function. In the remainder of the plots, the magnetic flux surfaces are shown at a single value of $t$ with the physical center of the plot coisciding with the vacuum-field magnetic axis. In plotg of the equilibrium quantities, $D_{1}=0$ marks the vacuum-field magnetic axis.

The etfects of changing plasma pressure un the NP-3 configuration are shown in Fig. 2. The vacuum-lield magnetic surfaces are given in Fig. 2(a). The concentric circles are ceatered on the vacuum magcetic axis, which is shifted towards the curvature center of the torus a distance of $5 \varepsilon a_{p} / 2 \gamma^{2}=0.8 \mathrm{~cm}$ with respert to the geometric center of the toroidal field coils. The effects of increasing the pressure are shown in Fig. $2(b)$ with $\langle\beta\rangle=17 \%$ and in Fig. $2(c)$ 
with $\langle\beta\rangle=50 \%$.

The vacuum configuration for NP-4 is given is Fig. 3(a). The vacuum magnetic axis is shifted $2.4 \mathrm{~cm}$ with respect to the geometric center of the toroidal field coils. The effecis of pressure on the configuration are stown in Fig. 3(b) with $\langle\beta\rangle=17 \%$ and Fig. $3(\Omega)$ with $\langle\beta\rangle=33 \%$. As $\langle\beta\rangle$ increases, the entire plasma shifts away from the center of the torus and the Shafranov shift of the innermost surfaces is clearly seen. We note that the plasma can be recentered by applying an external vertical feld.

In all the results presented up to this point, the exponent $k$ in the pressure function Eq. (19) has been set equal to 2.0, corresponding to a parabolic distribution in $\Psi$. The effects of varying this exponent are shown in Fig. (4). Figure $4(a)$ gives $\ell$ and $V^{\prime}\left(\Psi_{\text {tor }}\right)$ as functions of $\Psi_{\text {pal }}$ for $k=1.5$ and Fig. $4(b)$ shows these functions for $k=3.0$. The average beta is $33 \%$ in both cases. The value of $t$ and $V^{\prime}$ on axis is the same for both values of $k$. For the steeper pressure profile, $k=3.0, V^{\prime}$ decreases rapidly near the axis and then becomes flat uear the piasma boundary. The shear in this case is strongeat near the axis.

In summary, our vacuum configuration has circular flux surfaces which are rigidly shifted towards the center of the torus due to the turoidal curvature. The rotational transform is constant for the vacuum case, with the same value $\iota=0.5$ obtained for both devices. Funato et af ${ }^{20}$ give some numerical and experimental results for the vacuum configuration of the NP-4 device. They find that the vacuum configuration formed by the circular solenoidal coils ronsists of nested circles with the magnetic axis shifted by about $1.6 \mathrm{~cm}$ from the geometric center of the toils towards the center of the torus. An electron beam metbod 
is used to meagure a mean rotational transfae? 1 per helical period, $\iota=0.7{ }^{20,21}$ which is approximately constant across the Bux surfaces. Their results are in qualitative agreement with those given here.

The primary effect of pregsure on the configuration is to induce a Shafranov shift, which produces a magnetic well and creates shear. At very high beta values, the entire plasma is shified away from the center of the torns, and an externa] Geld is required to keep the plasma ioside the vacuum vessel. The model predicts that the Asperator configurations are capable of achieving equilibrium beta values on the order of $(\beta\rangle=50 \%$. For a given beta value, the Shafranov shift is significantly less pronounced for NPP-3 than Sor NP-4. Trubaikor and Dabryakov $^{22}$ investigated plasma equilibrium in a stellarator whose magnetic axis is a (belically wrapped) geodesic on a torus, and found that if the inverse aspect ratio $\epsilon \leq 0.1$, a substantial decrease in the shift is obtained when the number of helical periods is increased from 8 to 16 . This agrees with our findings for NP-3 with 16 periods and NP-4 which has 8 belical periods.

\section{Discussion and Conclusions}

The primary focus of this work is on the development of an asymptotic technique for zeducing the equilibrium problem for a helical-axis stellarator to a two-dimensional Grad-Shafranov-type equation. The motivation for seeking this reviuction is to make use of the variety of analytic and numerical methods available for the analysis of two-dimensional MHD equilibria. There include efficient free-boundary equilibrium codes and a formalism for stability analysis using expangion tecbniques. ${ }^{23}$ In the case of the conventional stellarator, the 
interently three-dimensional equilibrium problem can be expressed to lowest order as a two-dimensional problem that has axisymmetry, with toroidal effects provided by a self-consistent determination of the Pfirsch-Schlüter surrentg. ${ }^{10}$ At first, it is not obvious that a similar aimplification procedure can be developed for a helical-axis stellarator. The key to solving the belical-axis problem is the introduction of a coordinate system. based on the characteristics of the lowestorder vacuum field lines. These characteristic coordinates assume the same role that the axisymuneiric coordinates assume in the case of the conventional stellarator. We note ithat the results of this study connect smoothly to those obtained in the standard stellarator expansion. ${ }^{10}$ Namely, the expression for the vacuum surface function $\Psi$ in the asynptotic limits of small $\Delta$ in the helical-axis expansion and small $\gamma$ in the standard expansion coincide when w : retsin both zeroth and first-order terms in the latter.

The helical-axis stellarator expansion provides a useful model of the Asperator NP-3 and NP-4 devices. The vacuum field predictions for the equilibrium properties are in qualitative agreement with other analyses. In this paper, we are able to estimate the effects of pressure on the equilibrium, while most previous studies concentrated on modeling the vacuum configurations. High-beta equilibria with significant stear and a magnetic well are predicted.

The ordering of the parameters chosen in this paper led to the result that the rotational transform over the torus is small, of order $\delta$, rather than finite as in the standard stellarator expansion. Because of this, we bad to order the pressure very small, $P \sim \delta^{\mathbf{4}}$. An alternate way to model the configuration would have been to increase the major radiug as we extond the length of the helical 
periad, which leads to the ordering

$$
\delta^{3} \sim \gamma^{3} \sim \epsilon \sim \beta \sim \sigma \ll 1 .
$$

Then, the transforra would be finite. The use of this alternate ordering would yield essentially the same results, with the exception that the toroidaly inward displacement $d$ of Eq. (13) would be absent. Based on previous studies, ${ }^{23}$ we believe that this ordering is the approriate one for stability investigations.

This study has much in coramon with the work of Hender and Carreras ${ }^{24}$ who also use a stellarator expansion to reduce the helical-axis stellarator equilibrium problem to two dimengions. Rather than modeling the helical fields explicitly by introducing an $\ell=1$ potential, they use a numerically determined vacuum field and work in a vacuum flux coordinate system. They have shown that the calculation of the equilibrium properties can be carried further than was done in our work, by determining the effects of the belical Parsch-Schlūter currents ag well as the torojdal ones. Their approach is particularjy appropriate for studies where accurate evaluation of the vacuum field has been obtained. Ourn provides a useful complement becaune it enables one to understand the contributions of the individual field components to the enuilibrium and stability properties of the system.

The introduction of characteristic coordinate may have more general applications. The operator $B \cdot \nabla$ reduces to a single partial derivative in these coordinates, and this perrnits integration of the magnetic differential equations which arise in the helical-axis stellarator-expansion formalism. We note that the B $\cdot \nabla$ operator appears often in MHD calculations, and other representations such as Hamada coordinates which serve to simplify this operator are often 
useful. A possible extension of this work would be to apply the stellaratorexpansion technique in characteristic coordinates to more complicated vacuum field geometries. This can be done even in the case where analytic expressions are not available for the characteristic coordinates, provided an equation for these coordinates can be integrated numerically. Hendor and Carrerag ${ }^{24}$ have successfully implemented this type of numerical technique in a vacuum fux coordinate system.

In summary, in this paper we have used atellarator-expansion techniques to provide an asymptotic model of stellaratora with a helical magnetic axis. We note that the characteristic coordinates necessary to represent the equilibrium solutions properly may bave more general uses, since they greatly simplify the B,$\nabla$ operator. We have used the helical-axis stellarator expansion in connection with an equilibrium computer code to estimate the equilibrium properties of two stellarators. The basic expansion seeng uteful in this context, and modifications of the basic expansion for the modeling of gystems with more complicated vacuun fields should be possible.

\section{Acknowledgments}

One of us (AEK) thanks Prof. M. D. İruskal for many helpful discussions, and for sponsorship under the Program in Applied Mathematicy at Princeton University. Additionally, we have both benefited from interaction with colleagues at Priaceton and elsewhere. This work was supported by the U. S. Department of Energy under Contract No. DE-AC02-76-CHO-3073. 


\section{REFERENCES}

${ }^{1}$ L. Spitzer, Jr., Phys. Fluids 1, 253 (1958).

${ }^{2}$ H. R. Koenig, U.S. Atsmic Energy Commission Rept. No. NYO-B05d (PM-S40, 1959).

${ }^{3}$ J. L. Johnson, Nucl. Txchnol./Fusion 2, 340 (1982).

A. F. Boozer, T. K. Chu, R. L. Dewar, H. P. Furth, J. A. Goree, J. L. Johtson, R. M. Kulsrud, D. A. Moaticello, G. Kuo-Petravic, G. V. Sheffield, S. Yoshikawa, and O. Betancourt, in Plasma Physica and Contralled Nuclear Fetaion Redearch (LAEA, Baltimore, 1982), Vol. 3, p. 129.

${ }^{5}$ J. P. Friedberg, Rev. Mod. Phys. 54, 801 (1982).

${ }^{-}$F. Bauer, O. Betancourt, and P. Garabedian, Magnetohydrodynamic Equilibrium and Stability of Stellarators (Springer-Verlag, New York, NY 1984),

7V. D. Shafranov, Phys. Fluids 26, 357 (1983).

${ }^{B}$ C. Mercier and H. Luc, Lectures in Plasma Physics (Commission of the European Communities, Luxembourg, 1974).

'S. Nagao, H. Chishma, and N. Sasaki, J. Phys. Snc. Japan 42, 1075 (1977).

${ }^{10} \mathrm{~J}$. M. Greene and J. L. Johnson, Phys. Fluids 4, 875 (1961).

${ }^{11}$ G. Anania, J. L. Johnson, and K. E. Weimer, Phys. Fluids 28, 2210 (1983).

${ }^{12}$ N. Krylov and N. Bogoliubov, Introduckion to Nonlinear Mechanics (Princetori University Press, Princeton, NJ 1947).

13M. D. Kruskal, in Mathematical Modelo in Physical Science, edited by S. Drobot (Prentice-Hall, Inc., Englewpod Cliffs, NJ 1963) p. 17.

${ }^{14}$ M. D. Kruekal and R. M. Kulsrud, Phys. Fluids 1, 265 (1958).

${ }^{15}$ J. L. Johnsan, H. E. Dalhed, J. M. Greene, R. C. Grimm, Y. Y. Hsieh, S. C. 
Jardin, J. Manickam, M. Okabayashi, R. G. Storer, A. M. M. Todd, D. E. Voss, and K. E. Weimer, J. Comput. Phys. 32, 212 (1979).

${ }^{10}$ A. E. Koniges, PhD Thesis, Princeton Univergity (1984).

${ }^{17}$ F. B. Hildebrand, Methode of Applied Mathematics (Prentice-Hall, Inc., Eraglewood Cliff, NJ 1965) p. 306.

${ }^{18}$ S. Nagao, H. Watanabe, Y. Funato, I. Sakamoto, N. Sasaki, and K. Nukui, Proceedings of the Seventh Symposium on Engineeting Problems of Fusion Research (Kooxville, TN 1977), Vol. 1, p. 841.

${ }^{19} \mathrm{~S}$. Nagao and Asperatur Group, International Workghop on Stellarators [Sch)oss Ringberg, F.R.G., 1980) Paper B-3-6.

${ }^{20}$ Y. Funato, I. Sakamoto, T. Takahashi, S. Kitajima, Y. Ikeda, M. Nakagawa, H. Watanabe, S. Nagao, and Asperator group, Proceedings of the Terth European Conferestee on Controlled Fusion and Plasma Physics (Mosccit, 1981), Vol. 1, p. 365 .

${ }^{21}$ Y. Funato, I. Sakamoto, T. Asaishi, T. Saito, and S. Nagao, Plagma Phys. 22, $545(1980)$.

${ }^{32}$ B. A. Trubnikov and A. V. Dobryakor, Sov. J. Plasma Phyg. 8, 29 (1982).

${ }^{23}$ G. Anania and J. L. Johnson, Phys. Fluids 26, 3070 (1983).

${ }^{24}$ T. C. Hender and B. A. Carreras, Phys. Fluids 27, 2101 (1984). 
TABLE I. Parameters for the Asperator NP Devices

\begin{tabular}{lll}
\hline Parameter & NP-3 & NP-4 \\
\hline Average major radiug $(R)$ & $80 \mathrm{~cm}$ & $152.4 \mathrm{~cm}$ \\
Minor radius $(a)$ & $3.5 \mathrm{~cm}$ & $13.3 \mathrm{~cm}$ \\
Bore radius of limiter $\left(a_{\mathrm{p}}\right)$ & $3.0 \mathrm{~cm}$ & $9.5 \mathrm{~cm}$ \\
Number of periods $(N)$ & 16 & 8 \\
Helical radius $\left(r_{0}\right)$ & $5.0 \mathrm{~cm}$ & $19.05 \mathrm{~cm}$ \\
$\Delta=r_{\mathrm{o}} / a_{\mathrm{p}}$ & 1.67 & 2.01 \\
$\epsilon=\mathrm{a}_{\mathrm{p}} / R$ & 0.038 & 0.062 \\
$\gamma=\mathrm{c} N$ & 0.60 & 0.50 \\
\hline
\end{tabular}




\section{FIGURES}

FIG. 1. Model equilibrium configuration of NP-3 for $\langle\beta\rangle=34 \%$. (a) Magnetic flux surfaces at $t=0, \pi / 2$, atd $\pi$. (b)e versus $\Psi_{\text {pol }}$. Darbed line is vacuum value. (c) $V^{\prime}\left(\Psi_{\text {tor }}\right)$ versus $\Psi_{\text {pol. }}$. Dashed line is vacuum value.

FIG. 2. Effect of pressure variation on the equilibrium properties of NP-3: flux surfaces, rotational transform $\iota$, and $V^{\prime}\left(\Psi_{\text {tor }}\right)$ with $(a)\langle\beta\rangle=0 \%,(b)$ $\langle\beta\rangle=17 \%$, and $(c)\langle\beta\rangle=50 \%$.

FIG. 3. Effect of pressure variation on the equilibrium properties of NP-4 with $(a)\langle\beta\rangle=0 \%,(b)\langle\beta\rangle=17 \%$, and $(c)\langle\beta\rangle=49 \%$.

FIG. 4. Equilibrium properties for NP-4 using different pressure function exponents: (a) $k=1.5$ and (b) $k=3.0$. The corresponding pressure function $P$ and the parallel current distribution $\sigma$ are also shown for each case. 

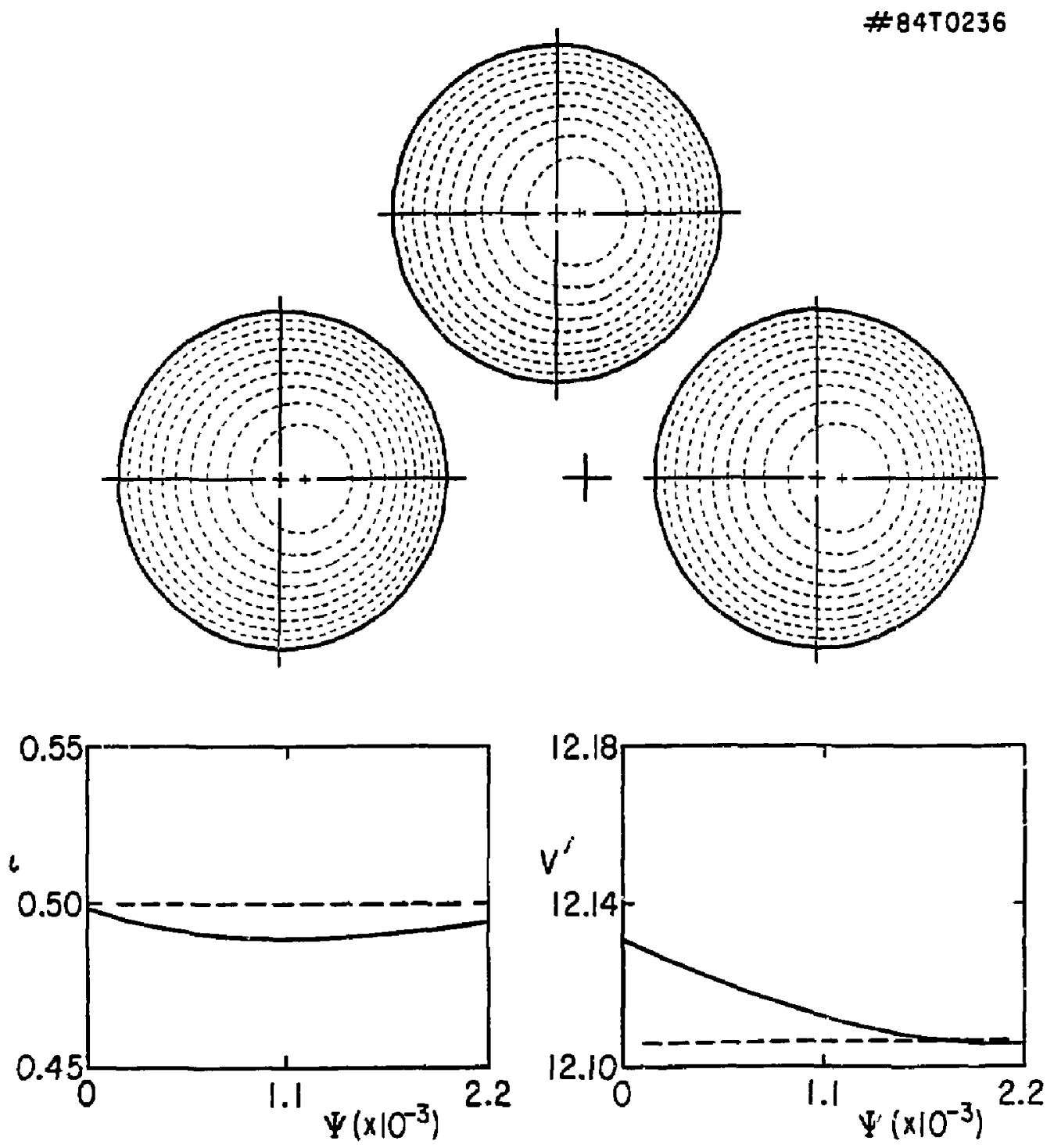

Fig. 1 
管
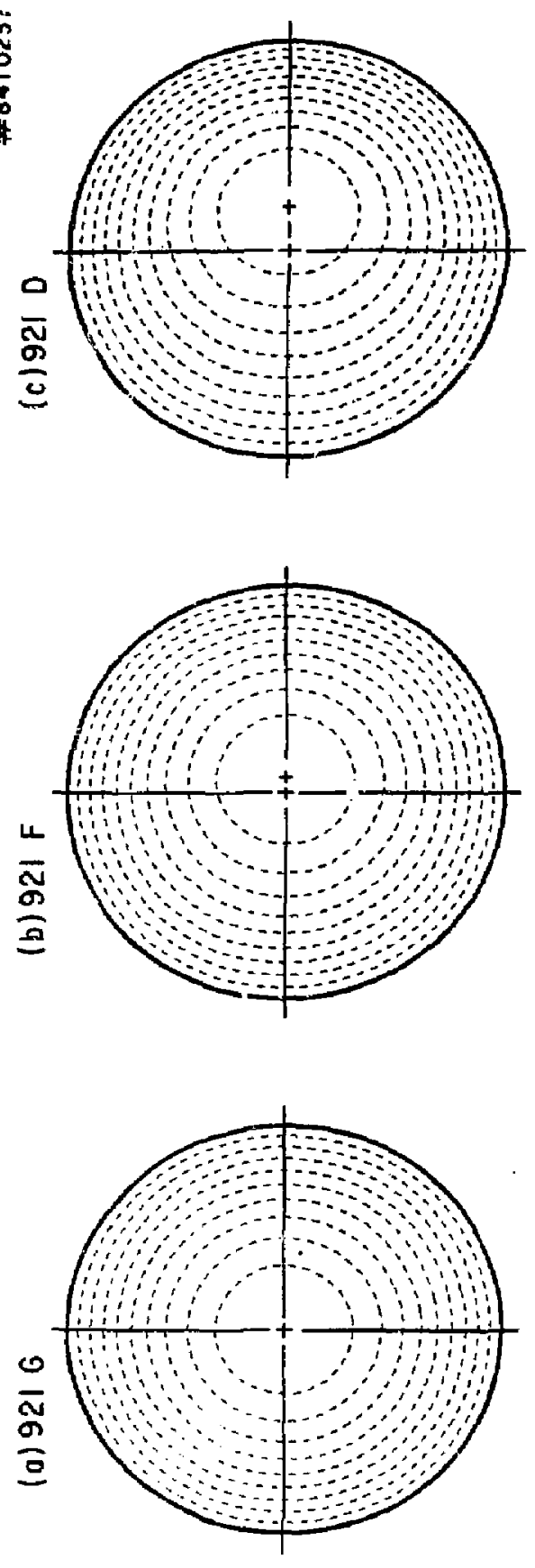
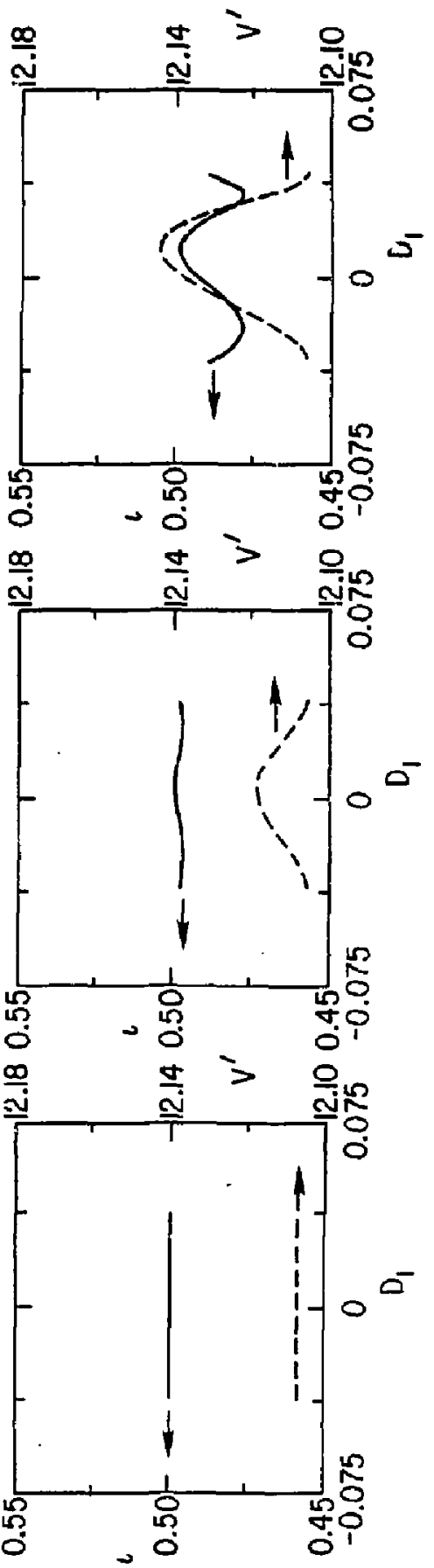


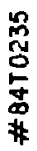
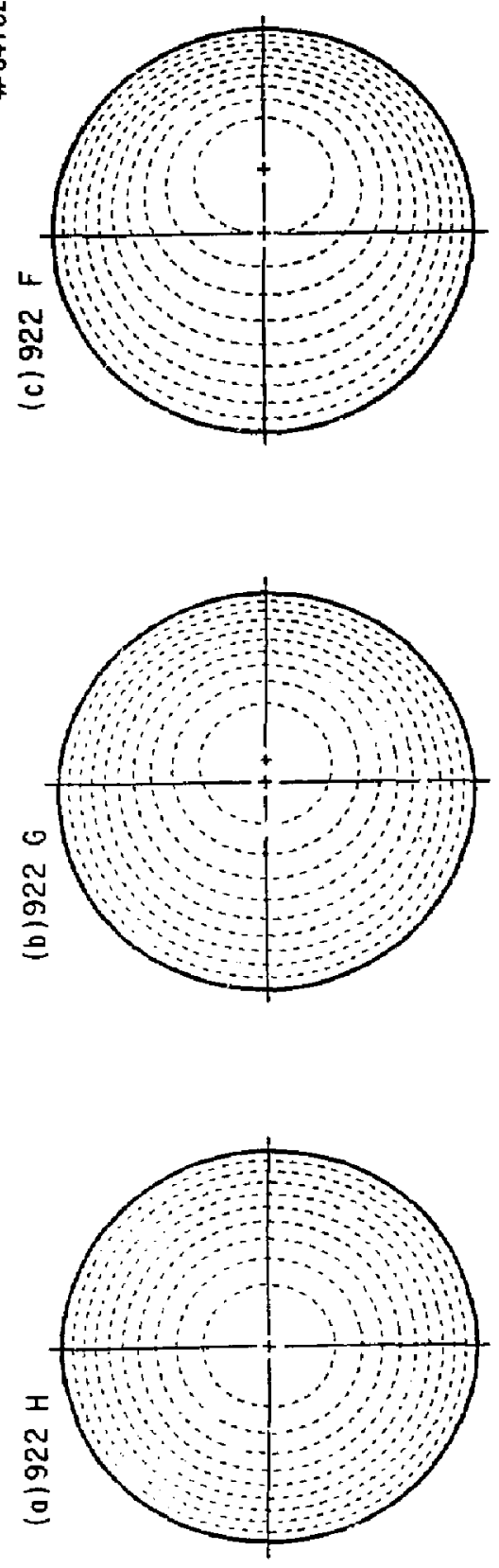
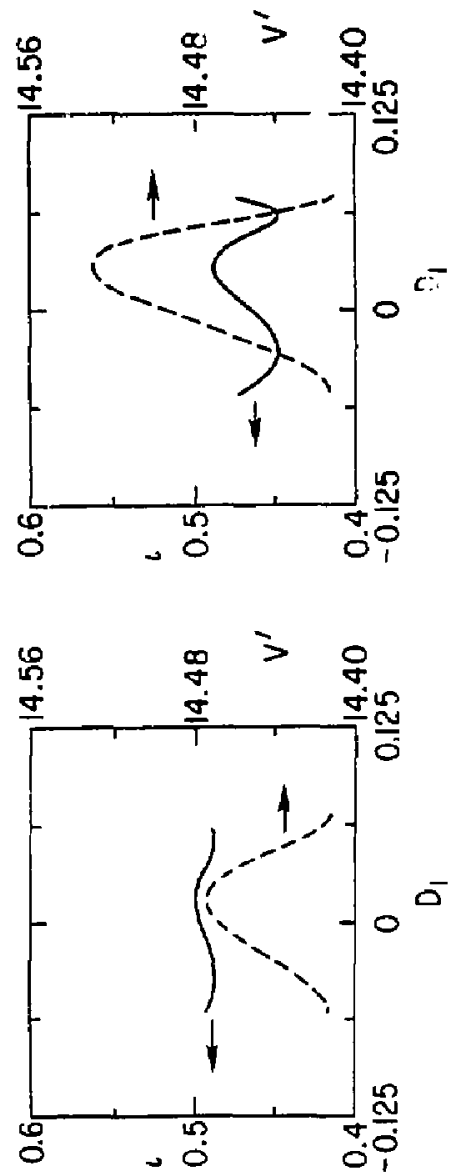

$\stackrel{5}{5}$ 
(a) 9220

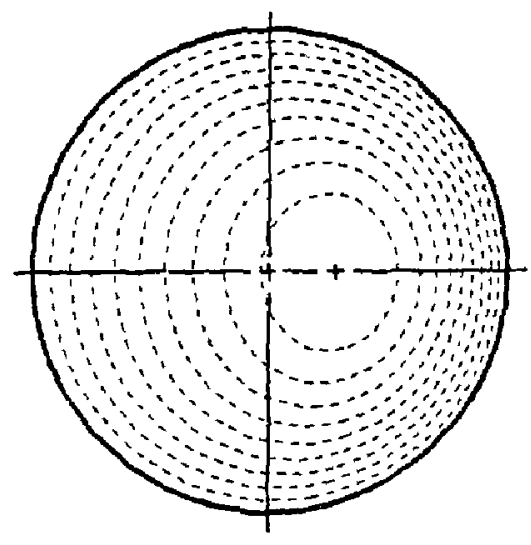

(b) $922 p$

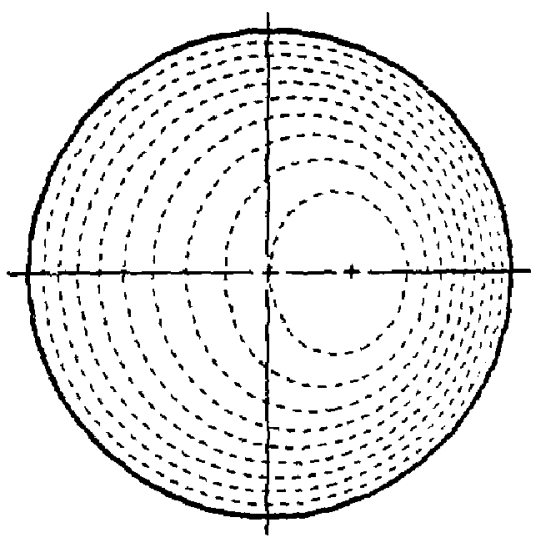

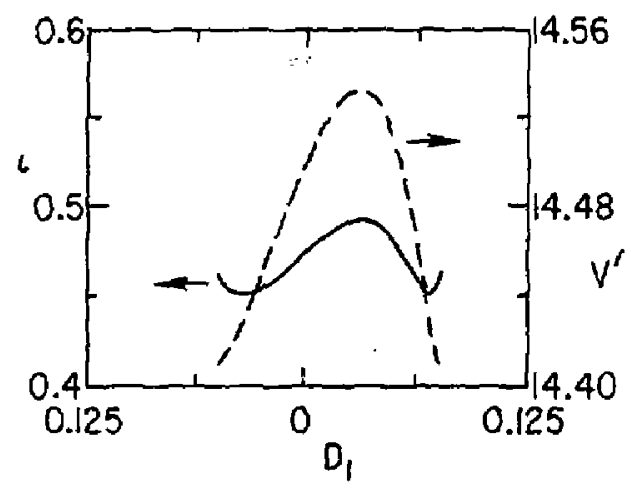
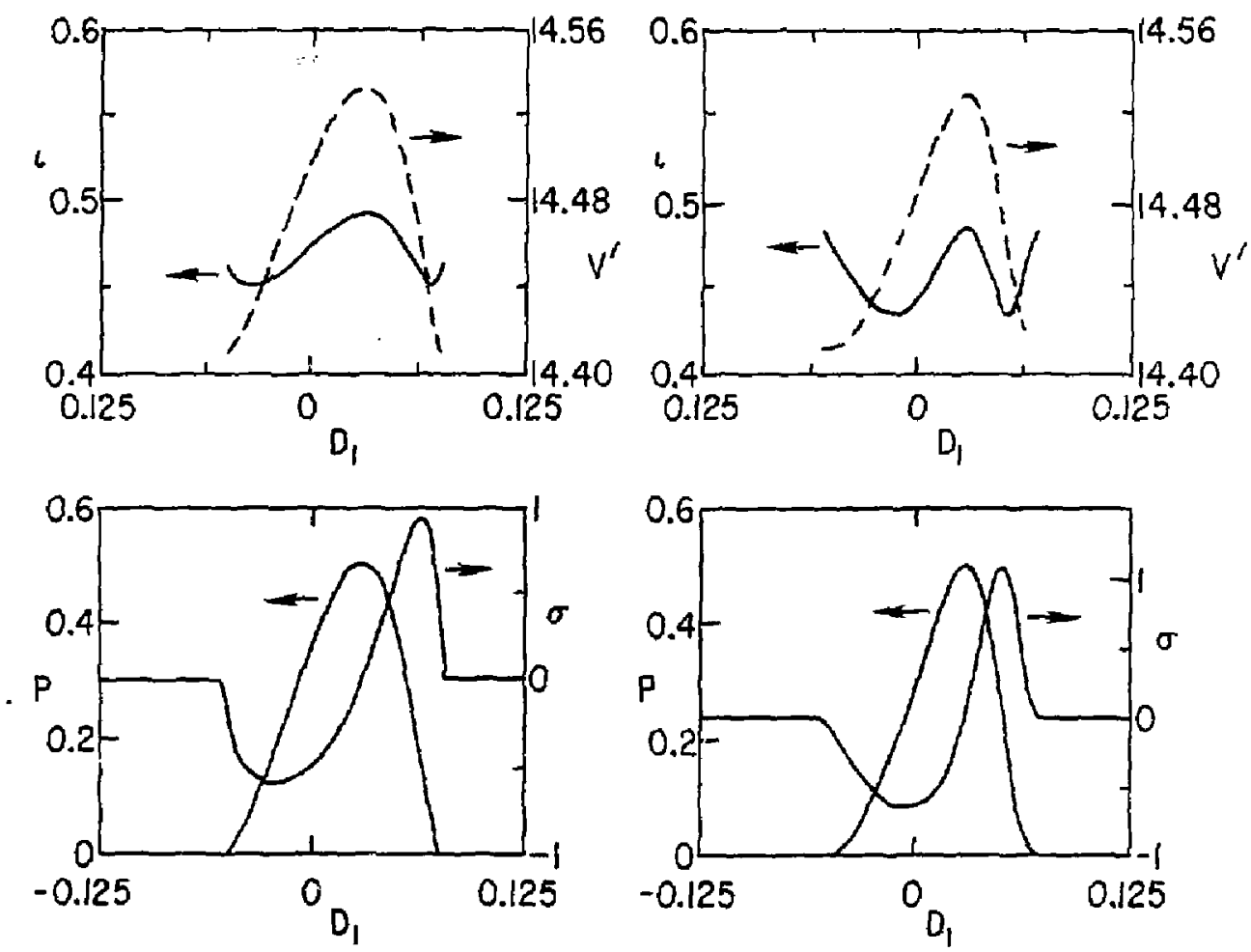

Fig. 4 


\section{REPRODUCED FROM \\ BEST AVAILABLE COPY}

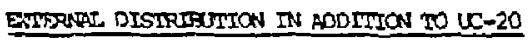

Plasina Res Lab, Austca Nat'l Ihiv, Alstrar.tA

DI. Trank J. Paloloni, Jniv of Wollongong, ALSTRALIA

Prof. I.R. Jones, Flinders Univ., ALSTRAIIA

Prof. M.H. Trennan, thiv Sydney, AJSTIFLIA

Prof. F. Cap, Inst Theo chys, Austrita

Prof. Saakk Verheest, Inst theotetische, ErronM

Dr. D. Palumbo, Xy XII Fusion Prog, BrIGIJM

Foole Foyale Mi li taire, Lab de phys Planas, BETGIOM

Dr. P.F, Sakanaka, Univ Astahal, ERATII

DT. C.R. Janes, Univ of Alberta, Cavad

Prof. J. Teichmam, Univ of Mantreal, CaNaDA

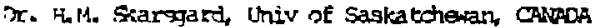

PTOE. S.R. Steetivasan, University of Calgary, CANADA

ProE, Tudor $\mathrm{N}$. Johnston, INRS-Energie, CANDDA

Tr. Hankes Bamarri, Univ Aritish Columibia, CanadA

DT. M.P. Bachynski, MPB 'nechnologies, Ine., FANDA

Chalk River, Whel Lab, CANaDA.

Thenow Li, SW I zst Physios, Chind

zibrary, Tsing the University, crma.

Librarian, Instibute of physics, CHDA

Inst Plasma Dhys, Academia sinica, Gran

ir. Peter Lukac, Komenskeho Univ, CandosianakA

The Librarian, Ouham Laboratory, enxifano

Prof. Schatzman, Observatoire de Nice, Francs:

J. Rader, CBLBPS, FFanCE

AM Dupas Library. Al Dupas Library, FRANC:

Dr. Iem thial, Academy sibliographic, have Karg

Probrint :ibrary, Cent Res Inst Phys, Hurafur

T. S.K. Treliz:, Panjah Uriversits; IDDIn

Dr. Irxara Wohan Lal Das, Banaras Hinah Univ, IDOIA

Dr. I.K. Chavda, South Gujarat Univ, INIA

Dr. R.K. Ghaj lani, vikram Oniv. IMDIA

วr. B. Dasqupta, Saha Inst, INDIA

Dr. D. Kas, Physical Research Lab, MDIA

$x$. Philitp Rosenalu, Isreel Inst Tech, IsRafr

Prof. A. Ouperman, Tel hviv thiversity, ISRAEL

Prof. G. Rostagni, Univ Di Padown, Fraty

Librarian, Int 1 ctrr theo Ehys, ITPLY

iniss Clelia je Pato, Assoe suratar-eNEA, ITALY

Biblioteca, del ORR ERATOM, IIFLY

DT. H. Yantat, Toshiha Res \& Dev, JAPIN

Direc. Dept Ig. Tokanak Dev. JMERI, IRPAN

Prof. itohyuki Inoue, reversity of Tokyo, JAPAN

Zesearch Info Center, Nagoja University. JAPAN

Posf. Kyoji Nishikana, Univ of Hinoshima, JAPAN

Prof. Sigeru Mori, JAERT, JAPAN

istibary, Kyoto University, JAPAN

Prof. tchiro Kawakani, Nihon Univ, TAPAN

Froi. Satosini Itoin, Kyustu University, JAPAN

$i$. D.I. Onoi, 2dv. Inst Sci \& Tech, KOREA

Ted Iffo Division, KAER, KOREA

zibliother, Eom-Inst voor Plama, MetrierLANDS
Brof. B.S. Liley, University of riaikzto, NEN 2EAris Exof. J.A.C. Cabral, Inst Suparior inch, FORTYXAz Dr. Oetavian Petais, ALI GJA University, ROMANA. Prof. M, A. Hellbert, University of Nacal, SO AFRICA Dr. Johan de Villiers, Plasna Physics, Nucor, 30 AFrica Fusion Div. Library, JEN, SPAM

Prot. Hars wilhelmson Chalmers univ Tecin, SinDPN

Dr. Iemart Stenflo, university of MEA, SMDEN

Library, Rojal Iist Tech, SHEDeN

Centre de Recherchesen, Ecole Polytech Fot, SuLIREIAND

Dr. V.T. Tolok, Khakkov Phys Tech Ins, ISSP,

DT. D.D. Ryutov, Siberian Acai Sci, USSR

Dr. G.A. Elisev, Kurchatov Instibute, ISST

DT. V.A. Glukhikh, Inst Electro-Physical, isSR

Institute Gen. Physics, rEs:

Prof. T.J.M Boyti, univ college N sales, waLCS

Dr. K. Schindlar, Buhr Universitac, w. GEPUivy

Nuclear Res Fstab, Julich LUd, W. GERMANY

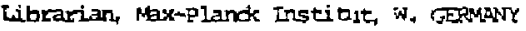

Bihliothek, Inst Plasmaforsciung, W. Jexukst

Prof. R.k. Janev, Tnst Phys, vuodolavi? 